\title{
Iso-Contour Queries and Gradient Descent with Guaranteed Delivery in Sensor Networks
}

\author{
Rik Sarkar* \\ Xianjin Zhu* \\ Jie Gao* \\ Leonidas J. Guibas \\ Joseph S. B. Mitchell ${ }^{\ddagger}$ \\ * Department of Computer Science, Stony Brook University. \{rik, xianjin, jgao\}@cs.sunysb.edu \\ $\dagger$ Department of Computer Science, Stanford University. guibas@cs.stanford.edu \\ $\ddagger$ Department of Applied Mathematics and Statistics, Stony Brook University.jsbm@ams.sunysb.edu
}

\begin{abstract}
We study the problem of data-driven routing and navigation in a distributed sensor network over a continuous scalar field. Specifically, we address the problem of searching for the collection of sensors with readings within a specified range. This is named the iso-contour query problem. We develop a gradient based routing scheme such that from any query node, the query message follows the signal field gradient or derived quantities and successfully discovers all iso-contours of interest. Due to the existence of local maxima and minima, the guaranteed delivery requires preprocessing of the signal field and the construction of a contour tree in a distributed fashion. Our approach has the following properties: (i) the gradient routing uses only local node information and its message complexity is close to optimal, as shown by simulations; (ii) the preprocessing message complexity is linear in the number of nodes and the storage requirement for each node is a small constant. The same preprocessing also facilitates route computation between any pair of nodes where the the route lies within any user supplied range of values.
\end{abstract}

\section{INTRODUCTION}

Wireless sensor networks have shown great potential for providing dense monitoring and sensing capabilities with modest cost and management effort. In many typical sensor network applications, sensors are densely deployed in a physical environment to provide good coverage at fine sensing resolutions. Existing work has established many fundamental mechanisms for sensor deployment to ensure coverage as well as energy efficient networking functions to collect data from these nodes.

There are two fundamental aspects of sensor networks that differentiate them from other types of wireless networks. First, it is the data from the sensor nodes, rather than the network nodes themselves, that is of most interest to the users. While many wireless networks, such as wireless LANs, cellular networks, and ad hoc mobile networks, focus on supporting lowlatency end-to-end communications and maximizing the system throughput, sensor network designs are often tailored towards their target application and are bound tightly to the physical environment that they are supposed to monitor/sense. In the most prevailing applications of environmental monitoring, sensors measure readings of the physical space, such as temperature, pressure, chemical concentration, and many others. Such physical quantities often exhibit continuity properties over space and/or time. Thus the smoothness of the physical signal field, and the spatial correlation of discrete sensor data, naturally suggest possibilities for data compression and exploitation for efficient system design.
A second unique property of sensor networks resides in their great potential in allowing seamless interaction between users and the physical world. In many civilian and military applications, the users operate in the same space in which the sensors are embedded. This allows novel applications in which real-time sensor data is quickly delivered to users of interest for appropriate response and actions. All of this eventually leads to a smart environment that could revolutionize the way we observe, interact with and influence the physical world.

In this paper we look at the iso-contours of a scalar signal field represented by sensor data, together with a local gradient routing scheme, with which the users can navigate in this signal field with guaranteed success.

Query for iso-contours of a signal field. Throughout the paper we assume the sensors are densely deployed in order to sample a continuous physical signal field. Indeed, there has been a large body of work on sensor deployment to ensure sensing coverage (see [1]-[4] and references therein). Thus the discrete sensor data allows for a close approximation of the underlying physical signal field. The signal field being monitored, such as a temperature field, chemical concentration map or traffic density follows physical laws and is naturally smooth with interesting spatial structures, such as the field iso-contours. For a continuous field, an iso-contour at an isovalue $x$ is the collection of points with value equal to $x$. In a discrete sensor network, this is often approximated by the collection of sensors with readings sufficiently close to $x$. The iso-contours thus identified help to describe and encode the regions with signal values within a specified range. For example, when the signal field is temperature, iso-contours may bound the 'hot' regions that indicate overheating or a fire. In another example of measuring chemical contamination, the iso-contours capture the polluted regions that require special treatment. The signal field can also be the energy map or traffic load on the networked sensors, and thus the iso-contours are related closely and provide information about the general health of the network or its traffic bottlenecks.

A few papers have studied compression, approximation and aggregation of iso-contours with space-efficient data structures, when sensors report their data along an aggregation structure to the base station [5]-[7]. In this paper we are interested in in-network data processing and the usage of iso-contours for navigation in the signal field. Consider a scenario in which 
sensors and users (such as rescuers or patrol officers) are embedded in the same physical space. Users carry hand-held devices and are able to directly communicate with nearby sensors. In particular, the users want to obtain directions to places that require attention or service, which are indicated by the sensor data being within a specified range. A naive solution is to flood from the query node the entire network, with all the nodes within the range responding to the query. This, however, can be quite energy expensive as many nodes not involved in the query will still be checked. Instead, we would like to quickly get the iso-contours of the limits of the query range and only report those nodes back to the user. These iso-contours bound the regions of interest, and may involve much fewer nodes than than all of the nodes within the query range. To abstract the problem, we want to support the following routing and navigation function with a low communication cost:

Iso-contour query: from a query node $q$, find the isocontours at value $x$. And its variations such as counting/reporting iso-contour components at given value/range.

Gradient descent routing. The most intuitive solution is to use gradient descent, by exploiting the natural continuity of the signal field. Starting from the query node $q$, the query message can be greedily guided either downhill or uphill, depending on the comparison of the value at $q$ and the target value $x$. This greedy descent routing is simple and requires only local knowledge. Thus it has been explored in a number of settings for low-cost data-centric routing [8]-[12]. Greedy descending/ascending can typically lead the query message to one iso-contour, unless the query message reaches a local minimum or local maximum, in which case the query gets stuck. Indeed, using simple gradient descent for an iso-contour query has a serious defect: the signal field may have multiple peaks and valleys, and greedy descending discovers at most one iso-contour, and is not able to discover all of the iso-contours due to the existence of local optima.

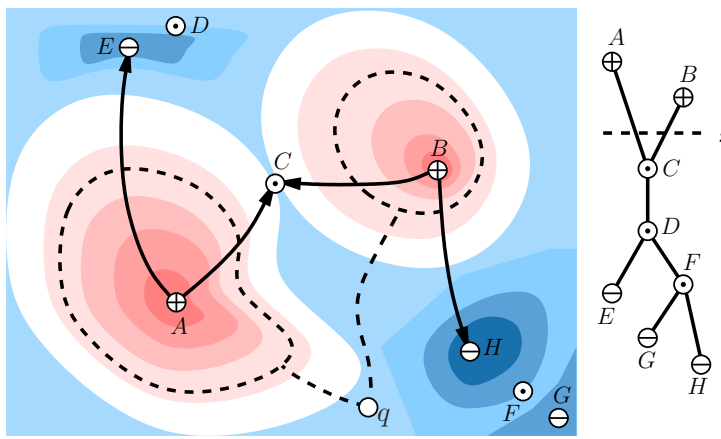

$\oplus$ local maximum $\ominus$ local minimum $\odot$ saddle point O query node $\rightarrow$ descending path - - - query trail

Fig. 1. The level sets of a signal field and the contour tree spanning all the critical points (in the right). The figure also shows some descending paths connecting the critical points.

Figure 1 shows an example of a potential field by drawing its level sets. Red colors mean hot and blue colors mean cold. We also show all the local maxima, minima and saddle points. A greedy gradient routing from a query node $q$ looking for a desired level contour will follow the local gradient and climb up the mountain. Once the query reaches the desired level it can locally trace out one contour, e.g, the contour on the left peak in the figure. However with only local information the query does not know whether there are other peaks and if so where they are.

The difficulty here is that the greedy gradient routing is completely local, while iso-contours reflect the global topology of the signal field. This is a general problem in navigation with a potential field, as has also been studied in robotics: with only information about the local potential one lacks the big picture of the signal field which is important for guaranteed success. In particular, the collection of critical points (local maxima, minima and saddle points) represents the global topology of the signal field. Thus, in order to make the local greedy descend algorithm always work, one needs to augment it with a compact representation of the critical points and their relationships.

Value restricted routing. A common practical problem is that of finding a path between given points such that everywhere in the path the value of some parameter lies within a given range. For example, finding a traffic free route between given souce and destination, or passing through a hilly region without climbing high mountains. The problem is also extremely relevant in communication networks where it is desirable to find paths that avoid congestion. As with iso-contours, the problem is of finding a globally optimal path, and requires knowledge of global interrelations between critical points.

Our contribution. We propose to investigate distributed algorithms to pre-process the iso-contour structures of the signal field by what is called the contour tree [13], using which a gradient routing scheme can successfully discover all iso-contours. In short, a contour tree is a tree on all the critical points of the signal filed and captures the topology of the iso-contours. It is a special case of the Reeb graph in Morse theory [15]. Take Figure 1 as an example, the right figure shows the topological contour tree consisting of eight vertices, corresponding to two local maxima, three local minima, and three saddle points. A contour tree captures how the connected components of the iso-contours merge/split as we increase/decrease the isovalue.

We propose an algorithm for the construction of the contour tree in a fully distributed manner. The basic idea is similar to the centralized construction [13], [16]-[18]. But we need to account for numerous robustness issues due to local noise and degeneracies, and lack of global coordination. We use distributed sweeps [19], initiated at local maxima and minima to identify the saddle points and nodes on the saddle contour. Next an information dissemination phase following the contour tree structure distributes necessary information for gradient routing. The same contour tree also permits a scheme for restricted value routing, and a labeling scheme such that validity of a restricted value route request can be determined simply from the labels of the source and destination nodes. The preprocessing involves all together four rounds of sweeps of the signal field and has a linear message complexity.

The invariant we maintain on a node $p$ is the $\max / \mathrm{min}$ 
value in the interior and exterior of the iso-contour component through each point $p$. This represents only a small constant storage requirement at each node. The gradient routing alternates between two operations (i) at a node on some saddle contour, it checks the split/merged contours and send one or two (if necessary) messages to the new connected components. (ii) at other nodes, the query message either follows an iso-level or follows gradient ascending/descending path to reach the desired contour. The gradient routing only uses information stored at a node itself and every routing step is justified, in the sense that there will definitely be a contour discovered for each query message. Thus no effort is wasted. Our simulations show that the gradient routing achieves comparable message complexity, when compared with the minimum spanning tree covering the iso-contour components, which is at most twice the length of the minimum Steiner tree, the optimal solution if the global knowledge about the entire signal field were available.

Lastly we note here that in this paper we only consider a static signal field, because the problem for a static signal field is already quite challenging. In practice, as the signal evolves over time we can periodically execute the contour tree construction phase. The maintenance of the contour tree for a time-varying signal field will be future work.

\section{Contour Trees And Gradient Routing}

Given a continuous signal field $\mathcal{F}$, the iso-contour (aka. level sets) at an isovalue $x$ is the collection of points $p$ with value $\mathcal{F}(p)=x$. The iso-contour at a particular isovalue may have multiple connected components. We denote by $C$ one connected component of an iso-contour and by $C(p)$ the connected component containing node $p$.

As we decrease the isovalues from the global maximum to the global minimum, the connected components on the isocontours may merge together, split, emerge, or disappear. These changes happen at critical points, such as local minima, local maxima and saddle points. The contour tree captures such topological changes of the iso-contours. In a contour tree, each node corresponds to a critical point, and an arc in the contour tree connects two critical points. In particular, as we start from $\infty$ and decrease the isovalue,

- at a local maximum, a contour component emerges;

- at a local minimum, a contour component vanishes;

- at a saddle point, two contour components merge into one or one contour component splits into two (see Figure 2). In the first case, there are two branches of the iso-contour eminating from the saddle point, representing the two components. Such a saddle point is called a merge saddle (with respect to decreasing isovalues). The second case with respect to decreasing isovalues is called a split saddle. (For increasing isovalues, split saddles and merge saddles are interchanged.)

It has been proved that the contour tree is indeed a tree. Further, without degeneracy (no two saddle points have the same values), a local maximum or a local minimum has degree 1 ; a critical point has degree 3 . To visualize, we place the vertices of a contour tree, i.e., the critical points, at the height

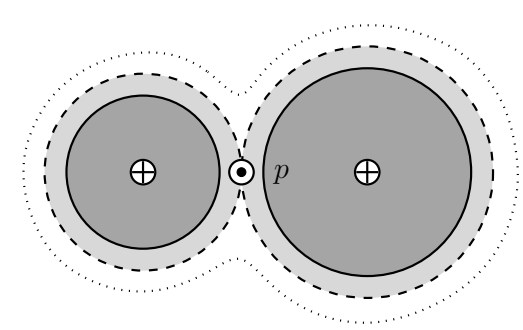

Fig. 2. $\oplus$ indicates a local maximum. $\ominus$ indicates a local minimum.

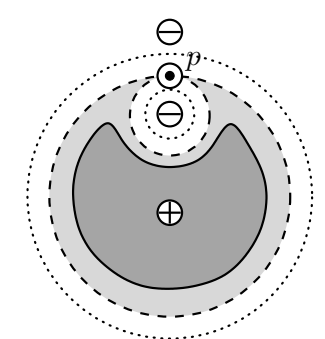

(ii) $\odot$ indicates a saddle point. Dark colors mean larger values. When we start from $\infty$ and decrease the isovalue, at a saddle point, (i) two contour components merge into one; (ii) one contour component splits into two.

levels of their values. A merge saddle has a ' $\mathrm{Y}$ ' shape and a split saddle has an inverted ' $\mathrm{Y}$ ' shape. An iso-contour is the level set by cutting the tree at the level of the isovalue. This possibly results in multiple contour components, with each component residing on an arc cut at that level. See Figure 1. Given a point $p$, if its contour component $C(p)$ stays on arc $\alpha$ of the contour tree, then we say $p$ is on $\operatorname{arc} \alpha$. In Figure 1, the iso-contour at the query value $x$ has two components, the left contour stays on the arc $A C$ and the right one stays on the arc $B C$.

Alternately, the contour tree can be thought of as a retract, as defined by $\mathcal{F}$, of the domain. The critical points of $\mathcal{F}$ are mapped to themselves as nodes of the contour tree. Continuous sets of contour components are mapped to edges of the tree continuously. Each edge is an open interval equal to the open interval of values at the corresponding sets of contour components, bounded by respective critical points, and is realized as an ascending/descending path between these critical points. If $\mathcal{R}$ is the map from domain of $\mathcal{F}$ to the tree, then under $\mathcal{R}$, a contour component is mapped to the point at intersection of itself and the path corresponding to the edge, it follows trvially that for any point $p, \mathcal{F}(\mathcal{R}(p))=\mathcal{F}(p)$.

Theorem 2.1. The contour tree computed from a morse function $\mathcal{F}$ is a retract of the domain of $\mathcal{F}$.

Sketch of proof. Take any open set $S$ in the tree. By the construction above, the pre-image $\mathcal{R}^{-1}(S)$ is an open set. Thus $\mathcal{R}$ is continuous, and by definition, it maps points on the tree to themselves, hence $\mathcal{R}$ is a retraction.

In a sensor network the continuous signal field is sampled by discrete sensors. Each sensor measures the value of $\mathcal{F}$ at its own location. Nearby sensors within transmission range can directly talk to each other. We would like to preprocess the signal field by computing the contour tree to facilitate gradient routing. This task faces the following challenges:

Local identification of critical points. In a continuous signal field, a critical point $p$ is a point with gradient 0 (all partial derivatives vanish at $p$ ). In a sensor network we can easily identify the local maxima and local minima. A local maximum has all the neighboring values no greater than itself. A local minimum has all the neighboring values no smaller than itself. However, it is not easy to identify saddle points, which have larger and smaller values in its neighborhood in an alternating 
way. When we do not have sensor locations or do not have accurate locations (say, the neighbors may switch their angular ordering), identifying a saddle point robustly is not straightforward. In our algorithm, the saddle points are discovered along with the construction of the contour tree, as the nodes where the contour components merge.

Distributed construction of the contour tree. The construction of the contour tree of a piecewise linear mesh has been studied before [13], [16]-[18], [20]. The best algorithm achieves a running time of $O(n \log n)$ on a piece-wise linear surface with $n$ vertices and can even be made to be output sensitive [17], [20]. However, these algorithms are centralized and are not appropriate for low-cost in-network processing in a distributed sensor network. We propose a distributed algorithm that involves four passes of sweeps, to be explained in details in subsection II-B. Thus the construction costs roughly $4 n$ message transmissions. After the preprocessing phase gradient routing with guaranteed success for iso-contour queries can be performed at any node in the network.

Handling noises and plateau regions. An important practical issue regarding contour trees for a sensor network is that the sensor data is a noisy approximation of the underlying smooth signal field, due to sensor innacuracy, hardware noise, etc. Thus there could be many more local maxima and minima in the sensor data than the the original (unknown, smooth) signal field. We propose two methods to handle this. First, we will locally simplify a contour tree by using topological persistence [21]. Small bumps will be chopped off. Second, we will not keep the entire contour tree at each node but rather only keep enough information for gradient routing. Thus, local optima due to noises in the measurement only influence a small neighborhood and are 'invisible' to queries from far away regions.

\section{A. Notations}

Before we describe the algorithm, we first state conceptually what we want to achieve with the contour tree construction and what we want to store at each node. An example of a contour tree is given in Figure 3 (i). A node $w$ on an arc $A B$ has a contour component $C(w)$ in between $C(A)$ and $C(B)$. The contour component $C(w)$ decomposes the entire signal field into two components, the interior and the exterior, corresponding to the two subtrees when edge $A B$ is removed. The interior contains the critical point $A$, which is reachable from $C(w)$ via a gradient ascending path. We call $A$ the ascending saddle. The exterior contains the critical point $B$, which is reachable by a gradient descending path. $B$ is called the descending saddle. Not every node have both ascending/descending saddles. Now

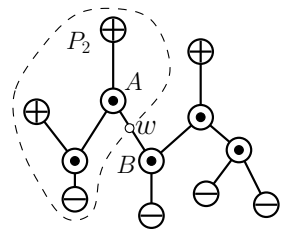

(i)

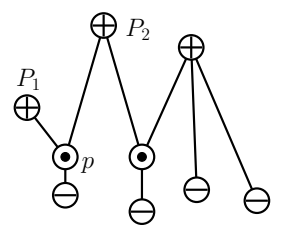

(ii)

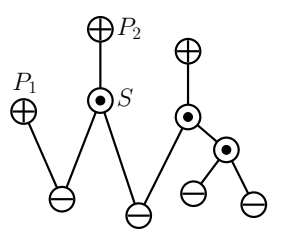

(iii)
Fig. 3. (i) A contour tree and the interior of $C(w)$ shown in the bounded region; (ii) merge tree; (iii) split tree. we will state the information stored at each node for gradient routing with success.

At a node $w$ (not on the contour component of a saddle), we will store four values:

- $I^{+}(w), I^{-}(w)$ correspond to the maximum and minimum value in the interior of $C(w)$;

- $E^{+}(w), E^{-}(w)$ correspond to the maximum and minimum value in the exterior of $C(w)$.

In addition, $w$ also keeps the node ID of the local max$\mathrm{ima} /$ minima it stores. This information is to guarantee that when we send a query message either uphill or downhill, we know for certain that there exist some contours for which we are looking.

For the consideration of network load balancing, each node also keeps information about the contours that split off/merge together at their ascending merge saddle or descending split saddle. Take saddle $A$ in Figure 3 (i) as an example. The contour component $C(A)$ is the union of two contours $C_{1}(A)$ and $C_{2}(A)$ that just merge together. Thus we keep at each node $u \in C(p)$, with $p$ on the arc $A P_{2}$ (with $A$ as a descending split saddle),

- the maximum/minimum values of the interior/exterior of both $C_{1}(p)$ and $C_{2}(p)$;

- gradient descending pointers leading to $C_{1}(p)$ or $C_{2}(p)$ or both.

This information helps us decide before we reach a saddle contour, whether it is worth visiting one or two of the contour components that split off of it and if so, how to get there.

To summarize, each node only keeps a small constant amount of information. Next we will explain how to get this information.

In the rest of this paper we assume a discrete network in which each node $u$ has an value $\mathcal{F}(u)$. The nodes have a communication graph $G$ that models the pairs of nodes who can directly communicate with each other. We assume no two sensors have the same values, if they do, ties are broken by their IDs (the one with higher ID is considered larger).

\section{$B$. Sweep to identify saddle points}

The construction of the contour tree and the spread of information about the peaks/valleys of the signal field are conducted by a sweep algorithm, similar to the one in [19]. Without loss of generality, we explain the details with the sweep top down. A node has its higher neighbors as the subset of neighbors with value strictly higher than itself, and its lower neighbors as the subset of neighbors with value strictly smaller than itself.

Each sweep is initiated and labeled by a critical node (a maximum, minimum or a saddle node). A node identifies itself as a local maximum if it discovers that all its 1-hop neighbors have value no greater than itself. It then initiates a sweep top down. The sweep algorithm runs in a distributed fashion on all the nodes. A node has two possible states, swept and not swept. Each local maximum node initializes itself as a swept node. When a node has all of its higher neighbors in the swept state, it changes itself to be swept. The nodes who participate 
in the sweep do not need to be synchronized. They decide on their own state and advance the sweep frontier with only local knowledge.

In the sweep initiated by a local maximum $p$, the sweep message carries the tuple $(p, \mathcal{F}(p))$, i.e., the node ID and value of $p$. Each node being swept will keep this information, as well as from which nodes it received this information. We define a descending path as a path in which each node has a value no greater than its precedent. During the sweep the information about a local maximum $p$ is propagated along descending paths from $p$. In addition, each node swept learns ascending pointers which eventually lead to the local maximum.

If a node gets two sweep messages from different local maxima, this indicates that two contour components start to merge. Thus a saddle should be identified. Since the nodes advance the sweep frontier in a distributed fashion, it may happen that two nodes at the same time both receive the sweep messages from two peaks. Thus we will need to define a saddle rigorously and resolve the ambiguity.

Definition 2.2. We define a node to be a merge saddle node if it is the one with highest iso-value with two descending paths from different critical points (other merge saddles or local maxima), i.e., it receives two sweep messages from different critical points.

Notice that this definition is recursive in nature. A merge saddle is precisely the first node when two contour components merge, as shown below.

Lemma 2.3. For a merge saddle $q$ of two critical nodes $p_{1}, p_{2}$, if we remove the sensors with values strictly smaller than $\mathcal{F}(q)$, and obtain a subgraph $G^{\prime}$, then $q$ is the cut node ${ }^{1}$ of $G^{\prime}$.

Proof: First, define $L_{1}\left(L_{2}\right)$ as the set of nodes in $G^{\prime}$ with ascending paths to $p_{1}\left(p_{2}\right)$. We claim that $L_{1}$ and $L_{2}$ has only node $q$ in common. If otherwise, $q \neq q^{\prime} \in L_{1} \cap L_{2}$. Since $q^{\prime}$ is a node in $G^{\prime}, q^{\prime}$ has a higher value than $q$. Now this contradicts with the definition that $q$ is the saddle node.

Now we argue that once $q$ is removed from $G^{\prime}$, then the set of nodes $L_{1}$ is disconnected from $L_{2}$. It will suffice to show that $q$ is a cut node of $G^{\prime}$. Now suppose that for two nodes $x_{1} \in L_{1}, x_{2} \in L_{2}$, there is a path $\mathcal{P}$ connecting them. This path $\mathcal{P}$ must use nodes other than those in $L_{1} \cup L_{2}$. Now take the first node on $\mathcal{P}$ coming out of $x_{1}$ that is not in $L_{1}$, denoted by $y_{1}$. Without loss of generality we can also assume that $y_{1}$ is just next to $x_{1}$ (otherwise take $x_{1}$ to be the preceding node of $\left.y_{1}\right)$. Now we must have $\mathcal{F}\left(y_{1}\right)>\mathcal{F}\left(x_{1}\right)$, since $y_{1}$ is not in $L_{1}$. Now take an ascending path from $y_{1}$, it will lead eventually to either a local maximum or a saddle, denoted by $p_{3}$. Thus the node $q$ cannot be a merge saddle with $p_{1}, p_{2}$, since there will be another saddle of $p_{1}$ and $p_{3}$, which is at least higher than node $y_{1}$. This shows a contradiction.

We also remark that with a top-down sweep we do not identify the saddle when one contour component splits into two - the split saddles will be discovered when we do a bottom-up sweep from local minima, in a completely symmetric fashion.

\footnotetext{
${ }^{1}$ Removing $q$ will result in two (or more) disconnected components.
}

Now we show how the merge saddle node is identified in a robust and efficient way. A node who is not a local minimum and first receives two sweep messages from different peaks $P_{1}, P_{2}$ will promote itself to be a potential merge saddle $S\left(P_{1}, P_{2}\right)$. In a distributed setting we need to worry about two issues: (i) two nodes $u, v$ (or more) may become potential merge saddles $S\left(P_{1}, P_{2}\right)$ for the same two peaks. In this case only the real saddle node (the one with highest isovalue) should survive. (ii) it may happen, if the sweep frontier does not proceed in the same speed, that the lower saddle may be discovered before the higher saddle. An example is shown in Figure 4. If the sweep from $P_{2}$ proceeds faster and reaches $S_{1}$ before it reaches $S_{2}$, then $S_{1}$ will notice it is a potential saddle for $S\left(P_{1}, P_{2}\right)$. The correct contour tree should have the saddle $S_{1}$ to be the merge saddle for $P_{1}, S_{2}$.

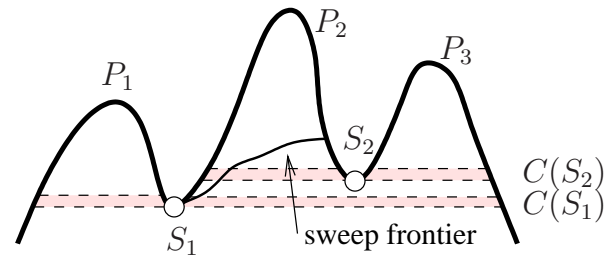

Fig. 4. If the sweep from $P_{2}$ proceeds faster and reaches $S_{1}$ before it reaches $S_{2}$, then $S_{1}$ will notice it is a potential saddle for $S\left(P_{1}, P_{2}\right)$. The correct contour tree should have the saddle $S_{1}$ to be the merge saddle for $P_{1}, S_{2}$.

The two problems will be resolved by the traversal of contour component, described below. Once a node $u$ becomes a potential saddle for two peaks $p_{1}, p_{2}$, it starts to traverse the contour component $C(u)$, defined as,

Definition 2.4. The contour component $C(u)$ for a node $u$ is defined as the set of nodes with isovalues equal or above $\mathcal{F}(u)$ and a lower neighbor (strictly) lower than $\mathcal{F}(u)$. If $u$ is a merge saddle for two critical points $p_{1}, p_{2}$, then $C(u)$ is partitioned into two components $C_{1}(u), C_{2}(u)$ (sharing only node $u$ ) that have ascending paths to $p_{1}, p_{2}$ respectively.

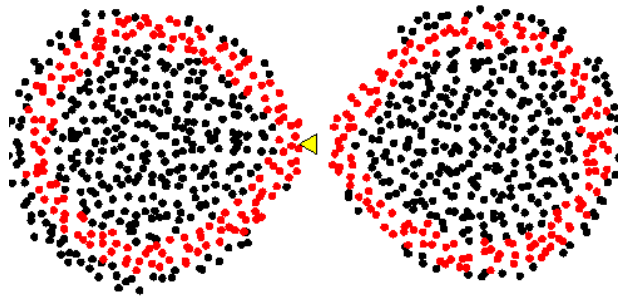

Fig. 5. A merge saddle (shown as the triangle) and its contour component (in red).

Thus a potential saddle node $u$ sends the tuple $\left(p_{1}, p_{2}\right)$ to the nodes in $C(u)$. To resolve the first issue that several potential saddle nodes compete for the real saddle, the traversal message from $u$ is suppressed if it hits a node $x$ with a traversal message from a winning potential saddle $u^{\prime}$ (with higher value) for the same two peaks. $x$ stops forwarding the message from $u$. Thus the traversal from $u$ will stop because it either visits all the nodes in $C(u)$ or if $u$ loses to some other potential saddle.

During the message dissemination, a backward pointer is cached at each node in the traversal. Thus a tree rooted 
at $u$, named $T(u)$, is established and used for information aggregation and for $u$ to learn about whether it wins and becomes the real saddle, or whether it loses the competition. In particular, a leaf $w$ in this aggregation tree will return to its parent 'loser' if $w$ has another winning traversal message, otherwise return the sweep message it has received. If a node is not yet swept, it waits for its sweep message before it returns information back. An interior node in the aggregation tree returns to its parent the union of the messages from its children. Now the potential saddle node $u$ becomes the real saddle for $p_{1}, p_{2}$, if (i) it does not get the 'loser' message from its aggregation tree; (ii) all the nodes in $C(u)$ are swept by either $p_{1}$ or $p_{2}$.

The new saddle $q$ will start with a new top-down sweep and they propagate the tuple $\left(q, \mathcal{F}(q), M\left(p_{1}, p_{2}\right)\right)$, where $M\left(p_{1}, p_{2}\right)$ indicates that $q$ is the merge saddle of two critical points $p_{1}, p_{2}$. All the nodes in $C(q)$ are considered swept by $q$ and the new sweep moves forward. We can also understand the sweep procedure by examining the contour tree. We can imagine a sweep initiated by a local maximum $p_{1}$ as moving down along the arc leaving $p_{1}$. At a merge saddle $q$, the sweep from $p_{1}$ meets a sweep from another local maximum $p_{2}$ (or from another merge saddle), and then the sweep moves down along the arc leaving the saddle $q$.

Notice that the sweep from a merge saddle $q$ is distinct from the sweeps from $p_{1}, p_{2}$. In fact, the merge saddle $q$ and all the nodes who receive the traversal message from $q$ do not forward the sweep from $p_{1}$ or $p_{2}$ anymore. In the case when a node $w$ has already forwarded the sweep from $p_{1}$ or $p_{2}$ by the time it gets the traversal message, it does not do anything and simply participates in the new sweep of $q$. If it gets the old sweep message from $p_{1}$ or $p_{2}$ after it has already started the sweep of $q$, it drops the old sweep messages. Notice that again we do not require synchronization. The old sweeps from $p_{1}$ and $p_{2}$ cannot propagate very far from $C(q)$, since $q$ stopped participating; thus, $q$ 's lower neighbors cannot possibly be swept, and so on and so forth.

If the merge saddle $q$ also happens to be a local minimum (in a setting with low discrete resolution), $q$ is in fact a merge saddle, a split saddle, and a local minimum all by itself. One trouble this may potentially cause is that the old sweeps from $p_{1}$ and $p_{2}$ may propagate without being dragged behind by $q$, since $q$ does not have lower neighbors. The system however, will eventually arrive at the correct state, since the sweep from saddle $q$ will overwrite the old sweeps from $p_{1}, p_{2}$. Later we will discuss mechanisms to deal with noises and plateaus to improve the efficiency.

The traversal also resolves the second issue mentioned above. In particular, $S_{1}$ cannot win before the saddle $S_{2}$ successfully identifies itself and proceeds with its sweep - this is because $S_{1}$ will only get its aggregated message when all the nodes in $C\left(S_{1}\right)$ have been swept, and $S_{2}$ and its descendants cannot be possibly swept before the saddle $S_{2}$ is done. During the aggregation phase for $S_{1}, S_{1}$ will learn about the sweep messages on $C\left(S_{1}\right)$. A subtle issue is that some nodes in
$C\left(S_{1}\right)$ may considered them swept by $P_{2}$ and report $P_{2}$ back to $S_{1}$. Thus $S_{1}$ learns that some of the nodes are swept by $S_{2}=M\left(P_{2}, P_{3}\right)$ and some nodes are swept by $P_{2}$ or $P_{3}$ alone. Now $S_{1}$ un-sweeps the nodes only swept by $P_{2}$ or $P_{3}$ and will only proceed to be the real saddle for $P_{1}, S_{2}$ when all the nodes are swept by $P_{1}, S_{2}$. In this case $S_{1}$ is initially proposed to be a saddle for $P_{1}, P_{2}$ but eventually becomes a saddle of $P_{1}, S_{2}$ when it wins.

To summarize, If there are two nodes both identifying themselves as a merge saddle, then the one with lower value will be swept and corrected (i.e., removed) eventually.

Lemma 2.5. With the algorithm above, there cannot be two nodes both identifying themselves as the merge saddle of two critical nodes. Thus the algorithm defines a unique contour tree structure.

After the top-down sweep, we have identified all merge saddles. By symmetry, we perform another sweep bottom-up initiated by local minima. Thus, after both sweeps we identify all saddle points and all nodes on the contour components of these saddles, thereby obtaining inherently the entire contour tree structure.

\section{Construction of the contour tree}

In this section we will extract the combinatorial contour tree after the saddles are identified. Notice that during top-down and bottom-up sweeps we have identified the merge tree (on all local maxima/minima and merge saddles) and the split tree (on all local minima/minima and split saddles). We will combine them to the contour tree such that each critical node learns its parent/child on the tree. Figure 3 (ii) (iii) shows the merge-tree and split tree.

We use gradient descending and ascending paths to discover the contour tree. Notice that all the nodes on the critical contours have been identified. Starting from a merge saddle $p=M\left(P_{1}, P_{2}\right)$, we follow gradient ascending paths towards $P_{1}, P_{2}$ respectively. If the ascending path towards $P_{1}$ reaches $P_{1}$ before it hits any other critical contour level, then $p$ will consider $P_{1}$ its parent in the contour tree. If the ascending path towards $P_{2}$ hits a split saddle contour $S$, then $p$ will consider $S$ as its other parent in the contour tree. Similarly $p$ also sends a descending path and identify its child in the contour tree. The operations for a split saddle, maximum/minimum are very similar, so we do not repeat here.

The operations require that an ascending path does not cross a split saddle contour without noticing it. This is guaranteed by the definition of a contour component. Suppose that in an ascending path $x$ has value $\mathcal{F}(x)<\mathcal{F}(q)$, with $q$ as a split saddle node, and the next node on the path $y$ has value $\mathcal{F}(y) \geq$ $\mathcal{F}(q)$. Thus $x$ must be on the saddle component $C(q)$, because $x$ has a value below $\mathcal{F}(q)$ and has a neighbor $y$ above it. This guarantees that the contour tree will be detected precisely as the combination of the merge tree and the split tree.

\section{Information dissemination}

With the contour tree constructed, we will need to disseminate information such that each node $w$ learns 
1) the maximum/minimum value, $I^{+}(w), I^{-}(w)$, inside the interior of its contour component $C(w)$;

2) the maximum/minimum value, $E^{+}(w), E^{-}(w)$, inside the exterior of $C(w)$.

This is done by information dissemination along the contour tree. By symmetry, we first explain how a node $w$ learns about the maximum value inside the interior/exterior of its contour component. Suppose that $w$ is on an arc $A B$. Recall that the interior of $C(w)$ corresponds to the subtree containing the ascending neighbor $A$, when $C(w)$ is removed. Thus the maximum of the exterior (interior) of $C(p)$ for a local minimum (maximum) $p$ is its own value.

We explain the basic operation by using the contour tree. For an $\operatorname{arc} e$, the removal of $w \in e$ leaves two subtrees $T_{1}$ and $T_{2}$, the maximum value in $T_{1}$ is sent through the arc, by a sweep, to $T_{2}$, and vice versa. In particular, we specify the dissemination rules at saddle points. See Figure 6. First, examine a merge

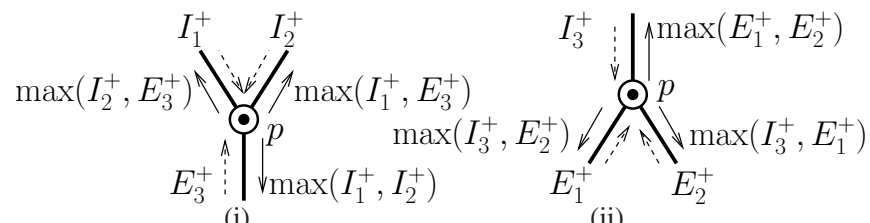

(i)

(ii)

Fig. 6. Information dissemination on (i) merge saddle; (ii) split saddle.

saddle $p$ with two incoming $\operatorname{arcs} e_{1}, e_{2}$ and one outgoing $\operatorname{arc} e_{3}$. Suppose by induction that the maximum is already learned and propagated along the $\operatorname{arcs} e_{1}, e_{2}, e_{3}$, as shown by $I_{1}^{+}, I_{2}^{+}, E_{3}^{+}$in the figure. In particular, say node $v$ on $e_{3}$ gets a message from a lower neighbor $u$ with the maximum of the exterior of $C(u)$, denoted by $E^{+}(u)$. Then node $v$ will update its maximum in the exterior of $C(v)$ as $\max \left(\mathcal{F}(v), E^{+}(u)\right)$.

In the same way, the maximum along $e_{1}, e_{2}, e_{3}$ arrives at the saddle contour $p$. Recall that the contour component $C(p)$ has two components $C_{1}(p), C_{2}(p)$, corresponding to the nodes with ascending paths along $e_{1}$ and $e_{2}$ respectively.

- for a node $w \in C_{1}(p), w$ sends $E_{1}^{+}=$ $\max \left(I_{2}^{+}, E_{3}^{+}, \mathcal{F}(w)\right)$ along the bottom-up sweep of $e_{1}$

- for a node $w \in C_{2}(p), w$ sends $E_{2}^{+}=$ $\max \left(I_{1}^{+}, E_{3}^{+}, \mathcal{F}(w)\right)$ along the bottom-up sweep of $e_{2}$;

- for a node $w \in C(p), w$ sends $I_{3}^{+}=\max \left(I_{1}^{+}, I_{2}^{+}\right)$along the top-down sweep of $e_{3}$.

This says that the nodes on $C(q)$ will initiate a sweep bottom-up along $e_{1}$ and $e_{2}$, and a top-down sweep along $e_{3}$ and propagate information as shown in Figure 6(i) accordingly. At a split saddle, information propagates in a similar way as shown in Figure 6(ii). We do not repeat here.

Notice that we do not need close synchronization among these sweeps. In particular, the bottom-up sweep on $e_{2}$ in Figure 6(i) can start when both $I_{1}^{+}$and $E_{3}^{+}$are done, even if the sweep $I_{2}^{+}$is not finished yet.

The information dissemination phase is initiated by the local minima and local maxima. A local minimum $p$ initiates a bottom-up sweep with value $E^{+}(p)=\mathcal{F}(p)$. A local maximum $p$ initiates a top-down sweep with value $I^{+}(p)=\mathcal{F}(p)$. Along each arc there are at most two sweeps in different directions. By symmetry, the information dissemination for the minimum value is done in a similarly way. In addition, the dissemination of both the minimum and the maximum can be integrated in the same sweep so that the total cost for this phase is roughly $2 n$.

In addition, we also disseminate for each merge (split) saddle $p$ with two parents (children) $p_{1}, p_{2}$, in a top-down (bottom-up) sweep from $C(p)$, information about $p_{1}, p_{2}$. At a merge saddle $p$, its contour component $C(p)$ has two parts, $C_{1}(p), C_{2}(p)$. Nodes on $C_{1}(p)\left(C_{2}(p)\right)$ have ascending paths to the parent $p_{1}\left(p_{2}\right)$. We are going to disseminate the ascending pointers along the sweep from $p$ downhill until the next critical contour. Specifically, each node on $C_{1}(p)$ records its hop count within $C_{1}(p)$ from the saddle $p$. This is called its index. For a node $w$, if $w$ has higher neighbors with ascending pointers to $p_{1}$, then $w$ has an ascending pointer to $p_{1}$ and its index is the minimum of the indices of those higher neighbors. A node may have ascending pointers to both $p_{1}, p_{2}$, for example, the saddle node $p$ itself and all the nodes with ascending paths to $p$. These nodes intuitively form the ridge in between the two mountains $p_{1}, p_{2}$. Similarly we disseminate the descending pointers along the ascending paths from a split saddle until the next critical contour. This information sweep can be combined with the previous sweep thus it does not incur extra cost. This information is used for the gradient routing to navigate in the field without always using the saddle node.

\section{E. Gradient Routing with Guaranteed Success}

The invariant we constructed so far enables an efficient gradient routing for iso-contour queries with guaranteed success. The gradient algorithm uses only the information stored at a node and its immediate neighbors.

Starting at $q$ we first check whether $x$ is beyond the range of the signal field, in which case we do not travel even one step and immediately return $\emptyset$. Effectively, this is by checking whether $I^{+}(q)<x$ and $E^{+}(q)<x$, or $I^{-}(q)>x$ and $E^{-}(q)>x$. If not, we know that there must be some nonempty iso-contours at level $x$ and we use a greedy gradient algorithm to find them. At the query node $q$,

- If $I^{+}(q) \geq x \geq I^{-}(q)$, then $q$ initiates a query message to follow the gradient uphill.

- If $E^{+}(q) \geq x \geq E^{-}(q)$, then $q$ initiates a query message to follow the gradient downhill.

We first explain the ascending query message from $q$. If a query message hits a node $w$ with isovalue $x$, it will then start a traversal along the contour component $C(w)$. This is done by the same algorithm as explained earlier. At the same time, we also need to check at $w$ whether it is worth getting even higher up - it is possible that at the interior of $C(w)$ there are still contours of value $x$. Again this is done by checking a higher neighbor of $w$, say $v$, whether $I^{+}(v) \geq x \geq I^{-}(v)$.

For an ascending query message at a node $w$, suppose $w$ stays on an arc with $p$ being an ascending merge saddle. Then we will check for two parents of $p$, denoted by $p_{1}, p_{2}$, whether 
we will need to ascend on one peak or both of them. Luckily this information has been disseminated for all the nodes on this arc. Thus $w$ will check the value range within the interior of $C_{1}(p), C_{2}(p)$ respectively. If the query value $x$ falls in the range, $w$ will initiate an ascending query message for it. See the red query in Figure 7 as an example of two query messages, one for each peak.

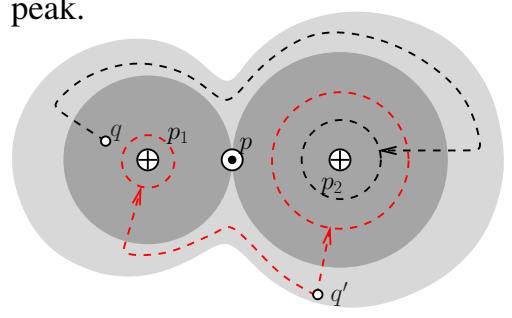

Fig. 7. Examples of two queries.

For an ascending query message towards say peak $p_{2}$, if $w$ has ascending pointers to $p_{2}$, this query message is simply delivered by gradient ascent routing, as the query from $q^{\prime}$ shown in Figure 7. If not, then the query message will follow a contour at a random value (below $\mathcal{F}(p)$ and above $\mathcal{F}(w)$ ) and follow the index-decreasing path, in order to cross the ridge and discover some ascending paths to $p_{1}$.

For a descending query message towards a merge saddle $p$ from one peak $p_{1}$, this query message will hit the saddle contour $C(p)$, from which it learns the value range outside $C(p)$ and the value range inside $C_{2}(p)$. Again dependent on the query value $x$, the query message may split into two, one for the exterior of $C(p)$ and continuing to go downhill, and one for the interior of $C_{2}(p)$ and going uphill. See the black query in Figure 7 for an example. The query looking for peak $p_{2}$, again, may not have an ascending pointer immediately. Then the query will take a random contour in between $C(p)$ and the lower critical contour, follow the index-decreasing direction until it discovers the ascending pointer to $p_{2}$. In both contourfollowing routing, we may go a random number of hops further after ascending pointers are discovered, in order to avoid always using the nodes on the ridge.

This two scenarios basically cover all the details of gradient routing. All other cases are symmetric and omitted here. The main idea is to send the query message along the contour tree, possibly splitting at internal branches, and discover all components of the iso-contour of interest. To summarize,

- The gradient routing algorithm is completely local and distributed and successfully finds all contour components at a given query level.

- Every step of the routing algorithm is justified, we send a query message only when we are sure there is something to be found. So no message will end up in vain.

- The routing scheme does not have to go through the saddles or follow critical contours, thus does not overload those nodes.

We note that this iso-contour query is the most basic query of a family of queries on iso-contours. Other iso-contour queries include: reporting the number of contours at value $x$, in particular, is there a single contour component? Range-limited queries (count/report contours within a value range)? These can be handled with either the iso-contour query as a subroutine, or by using a similar gradient routing algorithm. We omit the details here as the extension is relatively straight-forward.

\section{F. Value restricted routing}

In this section we consider the problem of finding paths within a restricted range of values in the network.

Routing request - $($ nodes $(s, t)$, range $[a, b]):$ Find a path $\mathcal{P}$ between nodes $s$ and $t$ such that at every node $x$ on $\mathcal{P}$, $a \leq \mathcal{F}(x) \leq b$, abbreviated as $a \leq \mathcal{F}(\mathcal{P}) \leq b$.

Keeping in mind that the contour tree is produced by a retraction $\mathcal{R}$ which maps every point $p$ and its contour component $C(p)$ to the same point $\mathcal{R}(p)$ in the contour tree, we describe the following results:

Lemma 2.6. For any path $\mathcal{P}$ between points $s$ and $t$, the image $\mathcal{R}(\mathcal{P})$ in the tree contains the unique path $\mathcal{P}^{\prime}$ in the tree between $\mathcal{R}(s)$ and $\mathcal{R}(t)$.

Proof: Since $\mathcal{R}$ is continuous, $\mathcal{R}(\mathcal{P})$ in the tree must be a connected graph spanning $\mathcal{R}(s)$ and $\mathcal{R}(t)$. Since $\mathcal{P}^{\prime}$ is the unique path between $s$ and $t, \mathcal{P}^{\prime} \subseteq \mathcal{R}(\mathcal{P})$.

Theorem 2.7. A suitable value restricted path exists in the network if and only if a corresponding path exists in the contour tree.

Proof: In the following, we assume $\mathcal{F}(s), \mathcal{F}(t) \in[a, b]$, since otherwise the request is clearly invalid.

A path in the contour tree implies a path in the network. Since the tree is a retract of the domain, a path $\mathcal{P}^{\prime}$ in the tree is also a path in the network. Also it is possible to traverse from $s$ to $\mathcal{R}(s)$ and from $t$ to $\mathcal{R}(t)$ along $C(s)$ and $C(t)$ respectively, without any alteration in $\mathcal{F}$. Appending these to $\mathcal{P}^{\prime}$ gives the required path.

A path in the network implies a path in the tree. Let us denote the known path in the network by $\mathcal{P}$. Let $\mathcal{P}^{\prime}$ be the unique path between $\mathcal{R}(s)$ and $\mathcal{R}(t)$. Then by lemma 2.6, $\mathcal{P}^{\prime} \subseteq \mathcal{R}(\mathcal{P})$. Since $\forall p, \mathcal{F}(\mathcal{R}(p))=\mathcal{F}(p)$, we have $\max \left(P^{\prime}\right) \leq \max (\mathcal{R}(\mathcal{P}))=\max (P)$ and $\min \left(P^{\prime}\right) \geq$ $\min (\mathcal{R}(\mathcal{P}))=\min (P)$.

The result above imples that on a value restricted routing request, we simply need to search the tree to verify if a suitable path exists and find one if it does. This theorem also suggests use of contour tree in finding connected components of ranges of iso-contours, which are harder to obtain than specific isocontours. According to the theorem above, these connected components correspond exactly to the connected components of the tree within the same range.

Lemma 2.6 suggests another method of finding value restricted routes. According to this lemma, the contour components on path $\mathcal{P}^{\prime}$ are ones that any path from $s$ to $t$ must intersect. In fact, these are exactly the contour components that separate $s$ from $t$ - with one inside and one outside. For any other component, both $s$ and $t$ lie on the same side, and hence can be avoided. In moving from $s$ to $t$ along any path, we can keep record of number of times each component appears, 
or simply push and pop them on a stack. The ones remaining in the stack at the end constitute the path $\mathcal{P}^{\prime}$. However, this method has the difficulty that there may be many components on the path and the stack can grow to be proportionally large.

We would thus like to stick to using theorem 2.7 to respond to the routing request, but without searching the tree every time. The theorem below shows that there is a compact labeling scheme that allows us to check and find a suitable route by simply comparing the labels.

Lemma 2.8. In any subtree $T^{\prime}$ of a contour tree there is a node whose removal divides the subtree into 3 connected components each containing at most $2 / 3$ the number of nodes in $T^{\prime}$.

Theorem 2.9. A contour tree admits a labeling scheme of label size at most $O(\log n)$, such that the range of values on the path between any two nodes in the tree can be derived from their labels.

Proof: Following lemma 2.8, recursively find the appropriate cut nodes...

\section{G. Handing noises}

With real sensor data, the signal field may have noises, causing lots of local optima. In practice we will de-noise the signal field by simplifying the contour tree during construction, to improve the construction efficiency. At a saddle node $q$, we will check the values of the two peaks $p_{1}, p_{2}$. Say $\mathcal{F}\left(p_{1}\right)>\mathcal{F}\left(p_{2}\right)$. If $\mathcal{F}\left(p_{2}\right)-\mathcal{F}(q)<\varepsilon$ and $q$ is at least $\gamma$ hops away from $p_{2}$, with $\varepsilon$ and $\gamma$ as upper bounds on the height and size of a bump to be considered as noises, we consider $p_{2}$ insignificant and chop it off. See Figure 8 (i). At the saddle $q, q$ will detect that $p_{2}$ is too small, thus it will be chopped at the value of $\mathcal{F}(q)$ and the sweep of $p_{1}$ will take over.

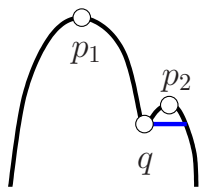

(i)

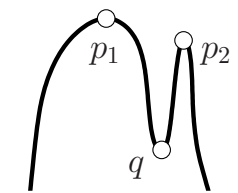

(ii)

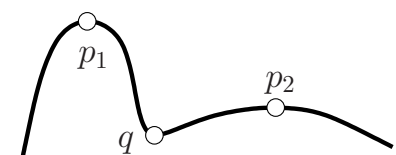

(iii)
Fig. 8. (i) a bump considered as noise and flattened; (ii) too high to be a noisy bump; (iii) too wide to be a noisy bump;

The above operations effectively 'smooth out' the signal field, guided by local geometric measures. This can substantially simplify the contour tree in a noisy data field. The gradient routing for iso-contour queries will miss at most some small components, whose sizes are controlled by $\varepsilon$ and $\gamma$.

\section{H. The presence of holes}

Throughout the paper we have mainly focused on a dense sensor field. When the network is sparse or has large holes, we will need to take special care of the holes. The major problem in the contour tree construction is that the contour component $C(p)$ for a saddle node may become disconnected. To deal with this, we propose two methods. For big holes (with diameter greater than $\delta$ ), we will explicitly detect such holes in a preprocessing step [22], such that the contour component hitting the hole boundary will be able to surround it and become connected. For the small holes (with diameter smaller than $\delta$ ), we will effectively search a wider band at contour traversal, i.e., the contour traversal message will be constrained as $\delta$ hops away from a node on the contour component, such that the small holes do not disconnect the contour component.

\section{Simulations}

We implemented the algorithm for constructing contour tree and for answering iso-contour queries with gradient routing. Our simulations do not take into consideration many important networking details, e.g., packet loss, delay and channel contention. This set of simulations is a proof of concept and aims to verify the correctness of the algorithm and evaluate the feasibility of the approach on the algorithmic level.

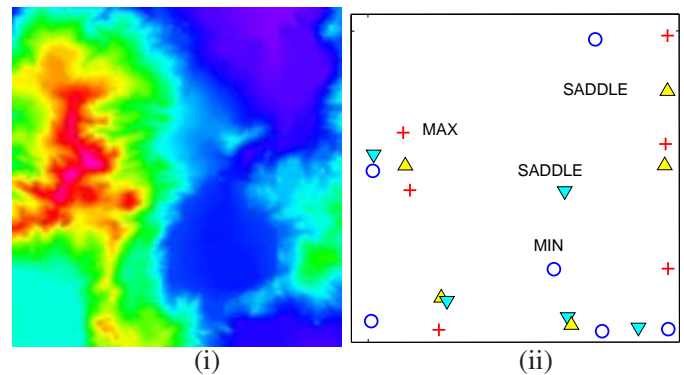

Fig. 9. (i) Elevation map of West Reno (obtained from usgs.gov). (ii) The critical points discovered by our contour tree algorithm with a 2500 node sampling.

Unless specified otherwise, the simulation setup consists of 1600 nodes, deployed in a 16 units by 16 units square region with unit disk graph as the communication model. Nodes are deployed in a perturbed-grid distribution, where each node is assigned a random position within its grid square. The average number of neighbors per node is about 21 . The sensors sample from a continuous signal field shown as in Figure 10 (i).

\section{A. Preprocessing cost for contour tree construction}

We first evaluate the cost of contour tree construction. We vary the number of nodes with the same signal field and count the total number of messages, assuming a broadcast medium. In our implementation, a random node on the sweep frontier is selected to become swept. The number of messages grows linearly in the number of nodes as shown in Figure 10 (ii). The constant factor is about $6 \sim 7$.

\section{B. Cost of iso-contour queries}

We compare the cost of gradient routing versus a global solution of using the minimum spanning tree to connect the query node $q$ and all the nodes on the iso-contour at value $x$, which is a 2-approximation of the minimum Steiner tree, the optimal (minimum cost) solution if the full knowledge of the signal field is available. We take 300 random queries with $q$ randomly selected within the field of deployment and the query value $x$ randomly chosen between the global minimum and global maximum values. For each query, we take the ratio of our query cost versus the cost of MST (both in terms of number of hops). We calculated the cumulative distribution, i.e., the percentage of queries for which the ratio is below $x$, in Figure 10 (iii). Roughly all cases have a ratio below 2 and $80 \%$ of the queries have a ratio below 1.4. 


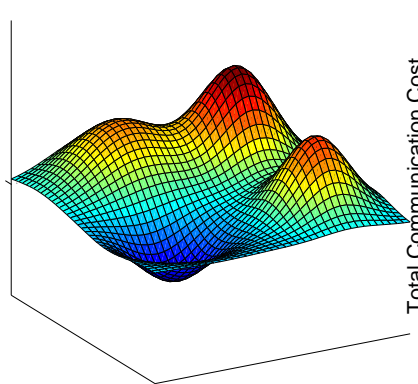

(i)

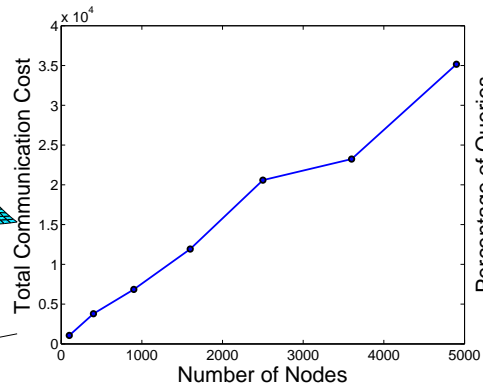

(ii)

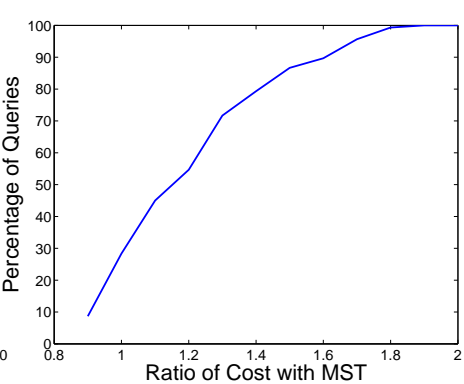

(iii)

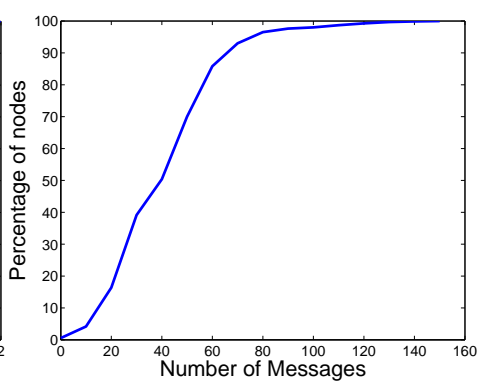

(iv)

Fig. 10. (i) The continuous signal field sampled by the distributed sensors; (ii) The message complexity of contour tree construction; (iii) The CDF of the ratio of our query cost v.s. the cost of MST; (iii) The CDF of the node load distribution.

\section{Load balancing}

In the same setup as the previous section, we plot the load on every node involved in the query procedure. A node is involved if it is on the routing path or is on the iso-contour to be queried. The maximum message load on any node is 148 , the average message load is about 48.7. The load distribution is shown in Figure 10 (iv), in which $90 \%$ of the nodes have a message load of below 70 .

\section{CONCLusion ANd Future Work}

In this paper we presented the distributed construction of a contour tree and its application in iso-contour queries by gradient routing with guaranteed delivery. Our future work is to update and maintain the contour tree for a time-varying signal field [23].

\section{REFERENCES}

[1] S. Meguerdichian, F. Koushanfar, M. Potkonjak, and M. B. Srivastava, "Coverage problems in wireless ad-hoc sensor networks," in Proc. of INFOCOM 2001, vol. 3, 2001, pp. 1380-1387.

[2] S. Kumar, T. H. Lai, and J. Balogh, "On $k$-coverage in a mostly sleeping sensor network," in MobiCom '04: Proceedings of the 10th annual international conference on Mobile computing and networking, 2004, pp. 144-158.

[3] X. Bai, S. Kuma, D. Xua, Z. Yun, and T. H. La, "Deploying wireless sensors to achieve both coverage and connectivity," in MobiHoc '06: Proceedings of the seventh ACM international symposium on Mobile ad hoc networking and computing, 2006, pp. 131-142.

[4] H. Zhang and J. C. Hou, "Maintaining sensing coverage and connectivity in large sensor networks," Wireless Ad Hoc and Sensor Networks: An International Journal, vol. 1, no. 1-2, pp. 89-123, January 2005.

[5] J. M. Hellerstein, W. Hong, S. Madden, and K. Stanek, "Beyond average: Toward sophisticated sensing with queries," in Proc. Information Processing in Sensor Networks (IPSN), April 2003, pp. 63-79.

[6] X. Meng, L. Li, T. Nandagopal, and S. Lu, "Contour maps: Monitoring and diagnosis in sensor networks," Computer Networks.

[7] S. Gandhi, J. Hershberger, and S. Suri, "Approximate isocontours and spatial summaries for sensor networks," in IPSN'07: Proceedings of the 6th international conference on Information processing in sensor networks. New York, NY, USA: ACM Press, 2007, pp. 400-409.

[8] M. Chu, H. Haussecker, and F. Zhao, "Scalable information-driven sensor querying and routing for ad hoc heterogeneous sensor networks," Int'l $J$. High Performance Computing Applications, vol. 16, no. 3, pp. 90-110, 2002.

[9] J. Liu, F. Zhao, and D. Petrovic, "Information-directed routing in ad hoc sensor networks," IEEE Journal on Selected Areas in Communications, vol. 23, no. 4, pp. 851-861, April 2005.

[10] J. Faruque and A. Helmy, "RUGGED: RoUting on finGerprint Gradients in sEnsor Networks," in IEEE Int'l Conf. on Pervasive Services (ICPS), July 2004.
[11] J. Faruque, K. Psounis, and A. Helmy, "Analysis of gradient-based routing protocols in sensor networks," in IEEE/ACM Int'l Conference on Distributed Computing in Sensor Systems (DCOSS), June 2005.

[12] F. Ye, G. Zhong, S. Lu, and L. Zhang, "GRAdient broadcast: A robust data delivery protocol for large scale sensor networks," ACM Wireless Networks (WINET), vol. 11, no. 2, March 2005.

[13] M. van Kreveld, R. van Oostrum, C. Bajaj, V. Pascucci, and D. Schikore, "Contour trees and small seed sets for isosurface traversal," in Proc. 13th Апnu. ACM Sympos. Comput. Geom., 1997, pp. 212-220.

[14] G. Reeb, "Sur les points singuliers d'une forme de pfaff complement integrable ou d'une fonction numerique," Comptes Rendus Acad. Sciences Paris, vol. 222, pp. 847-849, 1946.

[15] J. W. Milnor, Morse Theory. Princeton, NJ: Princeton University Press, 1963.

[16] M. de Berg and M. van Kreveld, "Trekking in the alps without freezing or getting tired," Algorithmica, vol. 18, pp. 306-323, 1997.

[17] H. Carr, J. Snoeyink, and U. Axen, "Computing contour trees in all dimensions," in Proceedings of the eleventh annual ACM-SIAM symposium on Discrete algorithms (SODA). Society for Industrial and Applied Mathematics, 2000, pp. 918-926.

[18] S. P. Tarasov and M. N. Vyalyi, "Construction of contour trees in 3D in $O(n \log n)$ steps," in $S C G$ '98: Proceedings of the fourteenth annual symposium on Computational geometry. New York, NY, USA: ACM Press, 1998, pp. 68-75.

[19] P. Skraba, Q. Fang, A. Nguyen, and L. Guibas, "Sweeps over wireless sensor networks," in IPSN '06: Proceedings of the fifth international conference on Information processing in sensor networks. New York, NY, USA: ACM Press, 2006, pp. 143-151.

[20] Y.-J. Chiang, T. Lenz, X. Lu, and G. Rote, "Simple and optimal outputsensitive construction of contour trees using monotone paths," Comput. Geom. Theory Appl., vol. 30, no. 2, pp. 165-195, 2005.

[21] H. Carr, J. Snoeyink, and M. van de Panne, "Simplifying flexible isosurfaces using local geometric measures," in VIS '04: Proceedings of the conference on Visualization '04. Washington, DC, USA: IEEE Computer Society, 2004, pp. 497-504.

[22] Y. Wang, J. Gao, and J. S. B. Mitchell, "Boundary recognition in sensor networks by topological methods," in Proc. of the ACM/IEEE International Conference on Mobile Computing and Networking (MobiCom), September 2006, pp. 122-133.

[23] H. Edelsbrunner, J. Harer, A. Mascarenhas, and V. Pascucci, "Timevarying reeb graphs for continuous space-time data," in SCG '04: Proceedings of the twentieth annual symposium on Computational geometry. New York, NY, USA: ACM Press, 2004, pp. 366-372. 